\title{
Honey bee flights under ethanol-exposure show changes in body and wing kinematics
}

Ishriak Ahmed ${ }^{1}$, Charles I. Abramson ${ }^{2}$, Imraan A. Faruque $^{1}$,

1 School of Mechanical and Aerospace Engineering, Oklahoma State University, Stillwater, Oklahoma, USA

${ }^{2}$ Laboratory of Comparative Psychology and Behavioral Biology, Oklahoma State University, Stillwater, Oklahoma, USA

* ishriak.ahmed@okstate.edu

Keywords: wing kinematics, insect flight, ethanol exposure, high speed tracking, body kinematics, flapping wing

\begin{abstract}
Flying social insects can provide models of the interactions needed for aerial robot swarms having limited processing resources. The ability to simultaneously make precise measurements of insect wing and body motions on such interacting insects is a recent capability, and chemical exposure may be used to modulate the interactions between insects. These interaction effects must be distinguished from the chemicals' effect on non-interacting insects. In this experiment, four high speed cameras (9000 fps) were used to track the wing and body motions of insects (Apis mellifera). Digitization, consisting of data association, hull reconstruction, and segmentation, achieved the first quantitative high speed measurements of ethanol exposed honey bees' wing and body motions. Kinematic analysis considered the trial wide mean and maximum values and gross wingstroke parameters, and tested deviations for statistical significance using Welch's t-test and Cohen's d test. The results indicate a decrease in maximal heading and pitch rates of the body, and that roll rate is affected at high concentrations (5\%). The wingstroke effects include a stroke frequency decrease, stroke amplitude increase, stroke inclination angle increase, and a more planar wingstroke. These effects due to ethanol exposure are valuable tools to separate from interaction effects.
\end{abstract}

\section{Introduction}

Individual insects flying in crowded assemblies perform complex aerial maneuvers by small changes in their wing motions. The complex behaviors and social interactions of honey bees (Apis mellifera) make them good candidates for quantifying the individual feedback rules that govern in-flight social interactions between animals. These mathematical rules may be a strong tool informing the design of autonomous aerial robotics swarm implementations on small, computationally-limited robotic platforms. Previous terrestrial experiments have demonstrated that the degree of honey bee social interaction (and hence these in-flight interactions) may be chemically manipulated through exposure to chemicals such as isopentyl acetate, ethanol, or pheremones such as 9ODA and 9HDA [1].

This study extends prior work on chemical exposure studies in 2D terrestrial locomotion to untethered flight of honey bees by examining the in-flight wing and body 
kinematics effects of ethanol treatment in honey bees (which have not yet previously been quantified), and by performing statistical analyses on these kinematics relative to unexposed agents. Nineteen motion variables are tracked for each case: 15 body states and 4 gross wingstroke parameters. The analysis approach tests mean and maximum values (computed over each trial) for statistical significance using Welch's t-test and Cohen's d test.

\section{Previous work}

Previous studies support the use of honey bees as a model for chemically-mediated social interactions. Bees engage in a wide range of simple and complex behaviors that include learning, communication. Honey bees foraging on fermenting nectar and fruit may naturally consume ethanol. While honey bees do not have a life stage dependent on alcohol (unlike fruit flies) [2], they readily self-administer high quantities and concentrations of alcohol [3] and demonstrate preferences for specific types of alcohol [4]. Bees and humans have been recorded exhibiting parallel aggression, locomotor, and learning changes following ethanol consumption [5,6]. Ethanol reduces the sting extension response threshold [6] and increases the number of stings [5]. High levels of exposure negatively impacts passive avoidance learning [7].

Locomotor activity decreases are dose-dependent [8], with small quantities inducing erratic movements [9] and high EtOH doses inducing decreases in both bee flight and walking activity $[3,10]$. Free flight foraging behaviors suggest the species can building ethanol tolerance [7].

Ethanol dose-dependent learning impairments have also been recorded in honey bees $[8,11,12]$, even in learning tasks as simple as association between an odor (conditioned stimulus) and a sucrose reward (unconditioned stimulus) in proboscis extension response (PER) experiments e.g. [8].

The previous work indicates that general honey bee behaviour is changed under ethanol influence and their flight behaviour may potentially be impacted as well. However, a review of archival literature shows that digitized recordings of in-flight wing and body motions for ethanol-exposed honey bees have not previously been reported. In this study, high speed visual tracking is used to measure body and wing motion states in flight after consumption of concentrations from $0 \%$ to $5 \%$, and statistical tests (Welch's t-test, Cohen's d effect size) are applied to those measurements to reveal these effects.

\section{Methods and approach}

\subsection{Experimental procedure}

Chemically-exposed honey bee preparation Foragers exiting a research hive were captured and anesthetized via storage below $0^{\circ} \mathrm{C}$ for 3 minutes and restrained in a harness made from a modified micro centrifuge tube. The insects were fed sucrose solution until no PER was present and let rest for approximately 24 hours at $22^{\circ} \mathrm{C}$. This preparation ensured a consistent metabolic state at the beginning of experiments [13] and minimized STRANGE effects [14]. A subset of insects were then fed sucrose-based solutions with varying ethanol concentration [8], kept for 15 minutes, and added to the flight test chamber. Each insect was removed from the test chamber less than 40 minutes after introduction to ensure flight is recorded under chemical influence.

High speed kinematics measurement A transparent T-shaped tunnel was attached to an Apis mellifera hive entrance with the two remaining exits exiting to 
outdoor space. Four Photron high speed cameras filmed the T-joint intersection at 9000 $\mathrm{Hz}$. The intersection was isolated with partitions in order to work as a confined 2337.80 in $^{3}$ test volume, with an 875.67 in $^{3}$ simultaneous capture volume. Recording was initiated manually when the insects started flying in the visible volume and ended when they left the volume covered by 3 or more cameras.

Digitizing tool Recorded insect flight trajectories were digitized using a high speed visual insect swarm tracker (Hi-VISTA) [15] implemented in MATLAB which can provide high-resolution tracking of multiple insects using a multiple camera system. The Hi-VISTA tracker takes synchronized frames from different cameras, identifies and removes background to recover multiple insect "blobs" which are then associated in different views. These insect targets reconstructed through voxel carving by checking consistency in views with the aid of camera projection matrices. Using the reconstructed insect visual hull, Hi-VISTA then segments the insects into wing and body and applies principal component analysis to vector geometry to determine their poses.

\subsection{Analysis}

Body parameters considered For this study, the state variables in each flight sequence are represented by 15 scalar variables. For a time history over $\left[0, T_{r}\right]$, where $T_{r}$ is the time length recorded, time $t$ was discretized as $t_{i}, i=1,2,3 \ldots, n$ at a constant sample frequency, and the mean value of a variable $h(t)$ measured the flight sequence was calculated as

$$
\bar{h}:=\frac{1}{n} \sum_{i=1}^{n} h\left(t_{i}\right), \quad t_{i} \in\left[0, T_{r}\right]
$$

and the maximum value is defined as

$$
h_{\max }:=\max _{t \in\left[0, T_{r}\right]}[h(t)] .
$$

Body parameters in each flight sequence was characterized by 13 scalar values as shown in Table. 1. We define the set of these scalars as $B$. Each of these variables are measured from the stability axes of the insect. For each $s \in B$ we consider population-wise mean and standard deviation.

The population mean value of a variable was defined as

$$
\mu(s):=\frac{1}{n} \sum_{i=1}^{n} s_{i} .
$$

where $n$ is the number of flight sequences recorded in the respective category $(0 \%, 1 \%, 2.5 \%, 5 \%$,$) . The population standard deviation of a variable was defined as$

$$
\sigma(s):=\left(\frac{1}{n} \sum_{i=1}^{n}\left(s_{i}-\mu(s)\right)^{2}\right)^{1 / 2}
$$

Wing parameters considered The insect wingstrokes were analyzed as a set $W$ comprised of 4 scalar variables.

The wing stroke, elevation, and pitch angles are represented as 3-1-2 Euler angles $(\phi, \psi, \alpha)$. Gross stroke frequency was determined by peak to peak time difference $T_{p}=1 / f$ in $\phi(t)$ of left wing. $\phi, \psi$ timehistories are then resampled to have a fixed number of discrete data points $N$ over each wingstroke. For each wingstroke the stroke plane angle $\beta$ and bias $\delta$ is determined by fitting

$$
\psi(k)=-\phi(k) \tan \beta+\delta, \quad k \in[1, N] .
$$




\begin{tabular}{|c|c|c|}
\hline Set & Notation & Description \\
\hline \multirow{13}{*}{$B$} & $\theta_{b}$ & Mean body pitch angle \\
\hline & $|\bar{u}|$ & Mean absolute forward speed \\
\hline & $|\bar{v}|$ & Mean absolute sideways speed \\
\hline & $|\bar{w}|$ & Mean absolute heave speed \\
\hline & $|\vec{p}|$ & Mean absolute roll rate \\
\hline & $|\bar{q}|$ & Mean absolute pitch rate \\
\hline & $|\vec{r}|$ & Mean absolute yaw rate \\
\hline & $|u|_{\max }$ & Maximum absolute forward speed \\
\hline & $|v|_{\max }$ & Maximum absolute sideways speed \\
\hline & $|w|_{\max }$ & Maximum absolute heave speed \\
\hline & $|p|_{\max }$ & Maximum absolute roll rate \\
\hline & $|q|_{\max }$ & Maximum absolute pitch rate \\
\hline & $|r|_{\max }$ & Maximum absolute yaw rate \\
\hline \multirow{4}{*}{$W$} & $f$ & Peak wingbeat frequency \\
\hline & $\Phi$ & Peak wingbeat amplitude \\
\hline & $\beta$ & Stroke plane angle \\
\hline & $\delta$ & Wingbeat shift \\
\hline
\end{tabular}

Table 1. Characterizing body $B$ and wing $W$ variables in flight sequence

$\beta$ can be used to compute planar motion of wing as in $[16,17]$

$$
\gamma(k)=\phi(k) \cos \beta-\psi(k) \sin \beta \quad k \in[1, N] .
$$

The stroke amplitude $\Phi$ for the wingstroke can be determined from the peak frequency of the Fourier transform of $\gamma$. Both wing motions were considered while determining $\beta, \delta, \Phi$ by concatenating the datapoints.

The population mean and standard deviation of these variables $s \in W$ are determined as

$$
\begin{gathered}
\mu(s):=\frac{1}{2 M} \sum_{i=1}^{2 M} s_{i} . \\
\sigma(s):=\left(\frac{1}{2 M} \sum_{i=1}^{2 M}\left(s_{i}-\mu(s)\right)^{2}\right)^{1 / 2} .
\end{gathered}
$$

where $M$ is the number of total wingstrokes recorded in the respective category $(0 \%$, $1 \%, 2.5 \%, 5 \%)$.

Statistical analysis tools In order to identify variables where data showed statistical differences, binary statistical analysis was applied by dividing the data in groups $\left(G_{1}: 0 \%, G_{2}: 1 \%, G_{3}: 2.5 \%, G_{4}: 5 \%, G_{5}: 1,2.5,5 \%\right)$.

The statistical tools applied to this dataset were Welch's t-test and Cohen's d test. Welch's t-test tests the null hypothesis that two populations have equal means for some variable. This hypothesis was tested for each $s \in S$ and $s \in W$ where the null hypothesis is

$$
\mu_{G_{1}}(s)=\mu_{G_{i}}(s), \quad i=2,3,4,5
$$

Welch's t-test does not assume equal variance and is helpful when sample sizes are not equal. $p$-values are used to indicate the probability of the null-hypothesis being true. Cohen's d quantifies effect size by $\mu(s)$ deviation in terms of pooled standard deviation. 
bioRxiv preprint doi: https://doi.org/10.1101/2022.01.18.476777; this version posted January 20, 2022. The copyright holder for this preprint (which was not certified by peer review) is the author/funder. All rights reserved. No reuse allowed without permission.

\begin{tabular}{|l|rr|rr|rr|rr|}
\hline Var $\backslash$ Mean/SD & $\mu_{\mathbf{0} \%}$ & $\sigma_{\mathbf{0} \%}$ & $\mu_{\mathbf{1} \%}$ & $\sigma_{\mathbf{1} \%}$ & $\mu_{\mathbf{2 . 5} \%}$ & $\sigma_{\mathbf{2 . 5} \%}$ & $\mu_{\mathbf{5} \%}$ & $\sigma_{\mathbf{5} \%}$ \\
\hline$\theta_{\underline{b}}(\mathrm{deg})$ & 38.33 & 8.43 & 30.78 & 10.47 & 40.18 & 15.79 & 44.55 & 8.51 \\
$|\bar{u}|(\mathrm{m} / \mathrm{s})$ & 0.30 & 0.08 & 0.32 & 0.08 & 0.28 & 0.10 & 0.34 & 0.17 \\
$|\bar{v}|(\mathrm{m} / \mathrm{s})$ & 0.19 & 0.05 & 0.17 & 0.07 & 0.14 & 0.09 & 0.21 & 0.10 \\
$|\bar{w}|(\mathrm{m} / \mathrm{s})$ & 0.12 & 0.03 & 0.17 & 0.09 & 0.16 & 0.13 & 0.16 & 0.06 \\
$|\underline{p}|(\mathrm{deg} / \mathrm{s})$ & 656.57 & 171.16 & 540.40 & 158.75 & 510.14 & 235.92 & 408.17 & 92.95 \\
$|\bar{q}|(\mathrm{deg} / \mathrm{s})$ & 226.93 & 104.92 & 131.17 & 61.97 & 159.95 & 94.17 & 188.87 & 57.16 \\
$|\bar{r}|(\mathrm{deg} / \mathrm{s})$ & 579.67 & 258.26 & 337.52 & 160.85 & 413.47 & 175.33 & 410.98 & 92.62 \\
$|u|_{\max }(\mathrm{m} / \mathrm{s})$ & 0.56 & 0.09 & 0.47 & 0.10 & 0.41 & 0.10 & 0.54 & 0.16 \\
$|v|_{\max }(\mathrm{m} / \mathrm{s})$ & 0.50 & 0.15 & 0.37 & 0.10 & 0.35 & 0.12 & 0.42 & 0.18 \\
$|w|_{\max }(\mathrm{m} / \mathrm{s})$ & 0.37 & 0.11 & 0.39 & 0.17 & 0.29 & 0.12 & 0.37 & 0.10 \\
$|p|_{\max }(\mathrm{deg} / \mathrm{s})$ & 3648.32 & 1391.59 & 3131.99 & 1847.50 & 2388.81 & 1465.98 & 1623.18 & 551.47 \\
$|q|_{\max }(\mathrm{deg} / \mathrm{s})$ & 1306.49 & 750.40 & 620.02 & 258.81 & 472.10 & 185.23 & 570.74 & 152.61 \\
$|r|_{\max }(\mathrm{deg} / \mathrm{s})$ & 3253.09 & 1784.58 & 1741.28 & 821.30 & 1699.60 & 574.15 & 1665.31 & 584.09 \\
\hline$f(\mathrm{~Hz})$ & 239.25 & 15.65 & 254.18 & 12.61 & 229.88 & 14.26 & 223.17 & 14.81 \\
$\Phi(\mathrm{deg})$ & 43.86 & 8.01 & 42.68 & 9.40 & 51.38 & 7.86 & 55.08 & 7.01 \\
$\beta(\mathrm{deg})$ & 27.41 & 9.60 & 31.40 & 5.91 & 33.99 & 7.35 & 33.70 & 6.80 \\
$\delta(\mathrm{deg})$ & 15.81 & 7.72 & 11.96 & 4.73 & 14.30 & 6.77 & 21.10 & 6.80 \\
\hline
\end{tabular}

Table 2. Mean $\mu_{i}$ and standard deviation $\sigma_{i}$ of $i=[0 \%, 1 \%, 2.5 \%, 5 \%]$ concentration

datasets.

\section{Results and discussion}

In this experiment we recorded flights in bees exposed with $20 \%$ sucrose and various ethyl alcohol concentrations (by volume): $0 \%$ (Control), 1\%, and 2.5\% and 5\%. 33 trials were collected.

The overall data used in this study is summarized in Table. 2, and the results of statistical tests are presented in Fig. 1 and 2.

\subsection{Body variable characteristics}

Pitch rates: $|\bar{q}|,|q|_{\max }$ Mean absolute pitch rates decreased in all percentages but the decrease is significant only in the $1 \%$ case. Maximum absolute pitch rate $|q|_{\max }$ is significantly reduced over all exposure levels. The changes in maximum pitch rates have a large Cohen-d effect size with $p<0.05$. A number of previous analyses indicate that airframe pitch modes are often unstable without neural feedback [18-20], this shift could signify that the pitch rate control mechanisms may have been affected and the unexposed insects flight envelopes include more aggressive motions.

Heading rate $|\bar{r}|,|r|_{\max }$ The overall decrease in mean absolute heading rates in all percentages is significant only in the $1 \%$ case. Maximum absolute heading rate $|r|_{\max }$ is significantly reduced over all exposure levels, with a large Cohen-d effect size (

$p<0.05)$. Previous work has indicated that "flapping counter-torque" provides passive stabilization through aerodynamic damping on this axis [21,22], suggesting the reduction in maximum heading rate may have different interactions with the underlying airframe relative to pitch rate.

Roll rate: $|\bar{p}|,|p|_{\max } \quad$ Both the maximum and mean absolute roll rates show that they are significantly reduced in the $5 \%$ case compared to control bees and not significantly in $1 \%$ and $2.5 \%$ groups. However, the overall comparison of control and exposed bees show reduction in both $|\bar{p}|,|p|_{\max }$. 

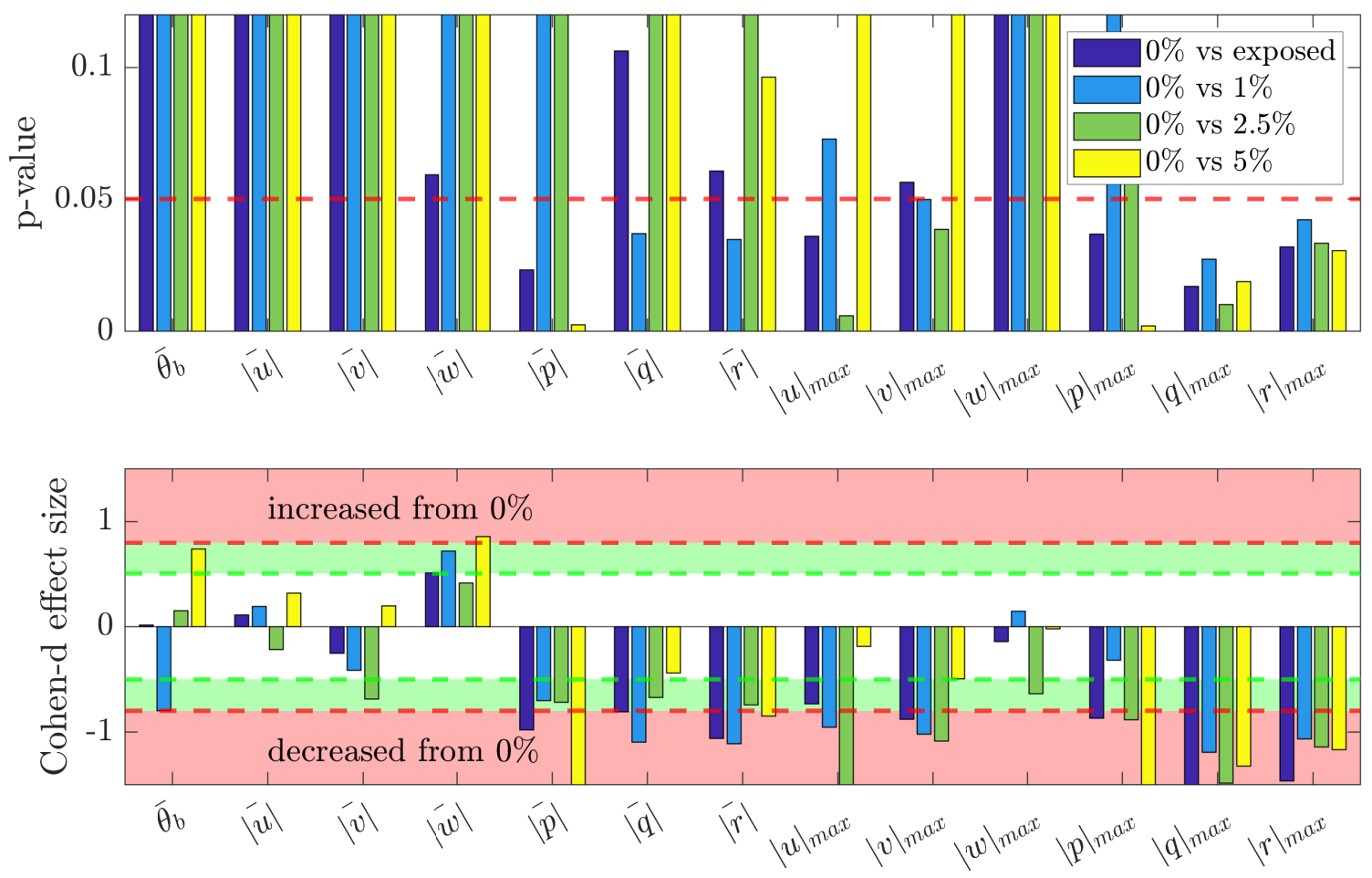

Figure 1. p values from Welch's t test and Cohen's d effect sizes are reported for the body variables in $B$. The comparisons are presented between the control honey bees $(0 \%)$ and either all exposed data groups $G_{5}(1 \%, 2.5 \%, 5 \%)$ and individual percentages in order to show which group is responsible for a statistically significant deviation from mean.

Body Speeds The mean body speeds are unaffected over the dataset in every comparisons. $|\bar{u}|_{\max }$ and $|\bar{v}|_{\max }$ had reduced in $2.5 \%$ to significantly affect the control vs exposed case. This trend is not continued in $5 \%$ case and cannot be conclusively linked to ethanol exposure.

\subsection{Gross wingstroke characteristics}

Insect asynchronous flight muscles generally operate near mechanical resonance [23] and frequency deviations often result in reduced performance. Hovering honey bees have primarily use relatively short amplitude and high frequency wingstrokes and maneuver via amplitude tuning [24,25]. Compared to control honey bees we see frequency increase and amplitude decrease in $1 \%$ group and frequency decrease with amplitude increase in 2.5 and $5 \%$. The $1 \%$ bees does not follow the trend of decreasing frequency with increased exposure level. Frequency decrease and amplitude increase was also observed in a separate dataset with manual determination of frequency by video footage observation [13].

Stroke plane inclination $\beta$ increased in all ethanol-exposed groups with a medium effect size. $\beta$ as a control input affects both forward flight speed $u$ and pitch rate $q$ [26]. Although increasing $\beta$ tends to increase forward speed, this effect can be mitigated by changes in flight force due to the frequency and amplitude changes. The wingstroke 

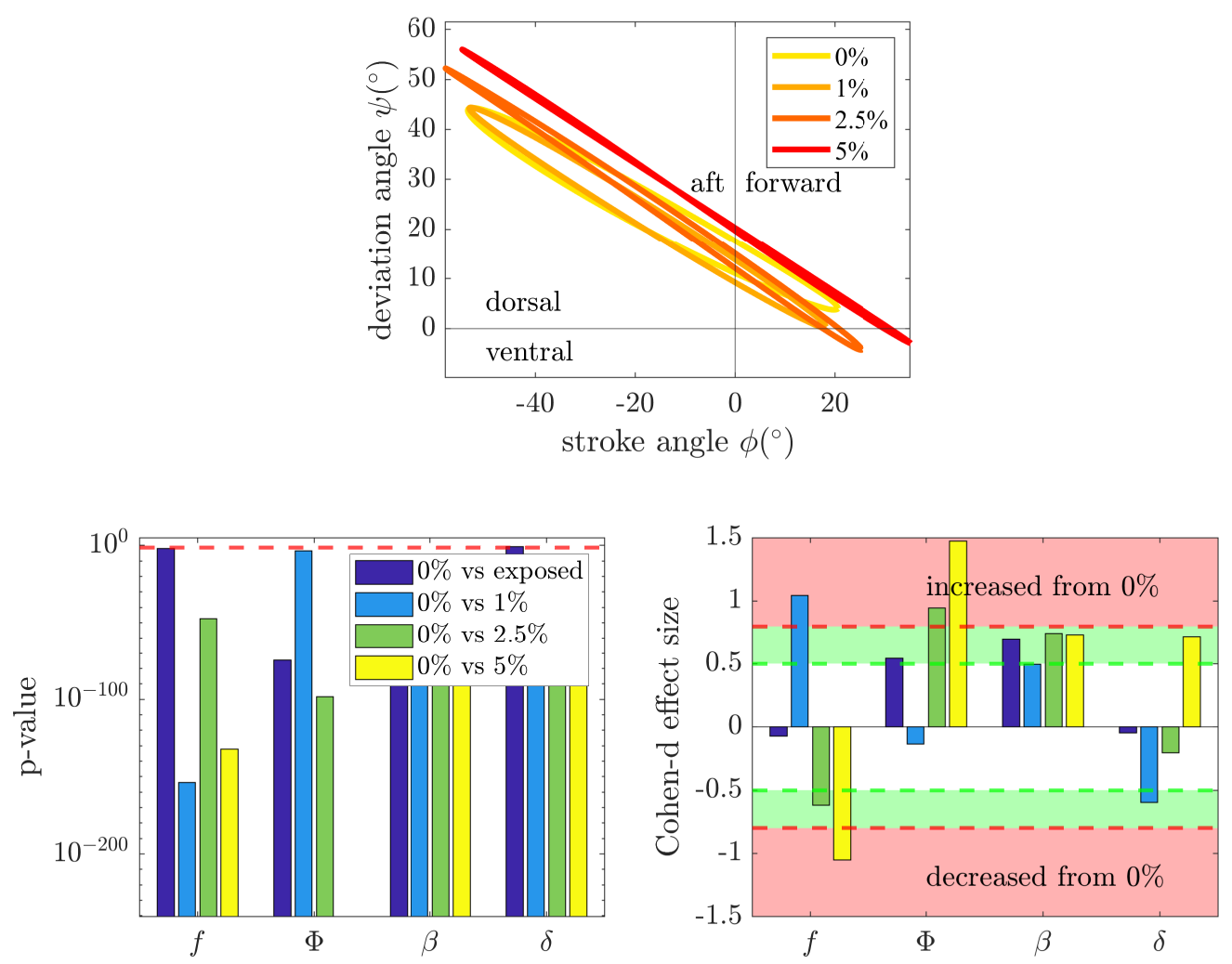

Figure 2. The mean wingstroke over all wingbeats are presented in the top which shows diminishing loop sizes in wingbeats with increased ethanol exposure. p values from Welch's t test and Cohen's d effect sizes are reported for the wing variables in $W$. The comparisons are presented between the control honey bees $(0 \%)$ and either all exposed data groups $G_{5}(1 \%, 2.5 \%, 5 \%)$ and individual percentages in order to show which group is responsible for a statistically significant deviation from mean. For all cases except for $\delta$ in $0 \%$ vs exposed, $(p<0.05)$

shift $\delta$ increased significantly only in the $5 \%$ group. A gradual mean loop size decrease with ethanol concentration (i.e., more planar wingstroke) is also visible in Fig. 2 (top). The mechanics and effect of non-planar wingstrokes are still not well understood $[17,27,28]$ and require further aerodynamic analysis.

\subsection{Limitations and observations}

Honey bees exposed to $10 \%$ ethanol solution did not initiate flight within 60 minutes in the test chamber and this study did not consider their flights. The $2.5 \%$ and $5 \%$ subjects initiated flight within 30 minutes of introduction to the test volume and displayed erratic ground movements prior to flight. Trials conducted at the $1 \%$ concentration in free-flight are quantitatively different from control insects and are distinct from the effects at higher concentrations, suggesting ethanol treatment effects are not a simple monotonic trend.

Statistical analyses such as these are limited to quantifying effects and the relative 
likelihood of such a measurement occurring due to chance. They do not identify the physiological or neural mechanisms behind such effects. This analysis also does not account for inter-dependence of variables. These are the first recorded quantitative high speed measurements of ethanol exposed honey bee flight, and experimental limitations on the number of animals constrain the dataset size. The differences were indicated as statistically significant and persisted when individual wingstrokes were analyzed (versus trial wide analysis), a combination which strengthens the study's applicability.

\section{Summary}

This paper presents the first quantitative high speed measurements of ethanol-exposed honey bee flight body and wing kinematic parameters. Kinematic changes induced by exposure to ethanol concentrations from $0 \%$ to $5 \%$ were studied using statistical analysis tools. The maximum heading and pitch rates reduce with increased ethanol exposure, while roll rates were affected at the $5 \%$ exposure level. Wingstroke analysis indicates a frequency decrease and amplitude increase for greater than $1 \%$ percentage exposure. Wingstroke loop size decreased and wing inclination angle increased with increased exposure level. Understanding the flight variables induced by this chemical manipulation in non-interacting flight conditions is an important result to distinguishing the effects of chemically mediated social interactions with neighboring flyers from chemical effects themselves.

\section{Acknowledgement}

This work was supported in part by ONR N00014-19-1-2216, NSF 1950805 and NSF 1743753.

\section{References}

1. Mumoki FN, Crewe RM. 20216 - pheromone communication in honey bees (apis mellifera). In: Blomquist GJ, Vogt RG (eds.), Insect Pheromone Biochemistry and Molecular Biology (Second Edition), pp. 183-204. London: Academic Press, second edition ed. (doi:https://doi.org/10.1016/B978-0-12-819628-1.00006-7).

2. Gibson J, May T, Wilks AV. 1981 Genetic variation at the alcohol dehydrogenase locus in drosophila melanogaster in relation to environmental variation: ethanol levels in breeding sites and allozyme frequencies. Oecologia 51, 2, 191-198.

3. Bozic J, DiCesare J, Wells H, Abramson CI. 2007 Ethanol levels in honeybee hemolymph resulting from alcohol ingestion. Alcohol 41, 4, 281-284.

4. Abramson CI, Sheridan A, Donohue D, Kandolf A, Božič J, Meyers JE, Benbassat D. 2004 Development of an ethanol model using social insects: Iii. preferences for ethanol solutions. Psychological reports 94, 1, 227-239.

5. Abramson CI, Place AJ, Aquino IS, Fernandez A. 2004 Development of an ethanol model using social insects: Iv. influence of ethanol on the aggression of africanized honey bees (apis mellifera 1.). Psychological reports 94, 3_suppl, $1107-1115$.

6. Giannoni-Guzmán MA, Giray T, Agosto-Rivera JL, Stevison BK, Freeman B, Ricci P, Brown EA, Abramson CI. 2014 Ethanol-induced effects on sting 
extension response and punishment learning in the western honey bee (apis mellifera). PloS one 9, 7, e100894.

7. Black TE, Stauch KLN, Wells H, Abramson CI. 2021 Effects of ethanol ingestion on aversive conditioning in honey bees (apis mellifera 1.). Journal of Comparative Psychology 135, 4, 559-567.

8. Abramson CI, Stone SM, Ortez RA, Luccardi A, Vann KL, Hanig KD, Rice J. 2000 The development of an ethanol model using social insects i: behavior studies of the honey bee (apis mellifera 1.). Alcoholism: Clinical and Experimental Research 24, 8, 1153-1166.

9. Mixson TA, Abramson CI, Božič J. 2010 The behavior and social communication of honey bees (apis mellifera carnica poll.) under the influence of alcohol. Psychological reports 106, 3, 701-717.

10. Maze IS, Wright GA, Mustard JA. 2006 Acute ethanol ingestion produces dose-dependent effects on motor behavior in the honey bee (apis mellifera). Journal of insect physiology 52, 11-12, 1243-1253.

11. Abramson CI, Sanderson C, Painter J, Barnett S, Wells H. 2005 Development of an ethanol model using social insects: V. honeybee foraging decisions under the influence of alcohol. Alcohol 36, 3, 187-193.

12. Abramson CI, Craig DPA, Varnon CA, Wells H. 2015 The effect of ethanol on reversal learning in honey bees (apis mellifera anatolica): Response inhibition in a social insect model. Alcohol 49, 3, 245-258.

13. Lane E, Faruque I. 2021 Insect in-flight kinematic adjustments during ethanol exposure. In: AIAA Scitech 2021 Forum, p. 0004.

14. Rutz C, Webster MM. 2021 Ethology adopts the strange framework for animal behaviour research, to improve reporting standards. Ethology 127, 2, 99-101. (doi:https://doi.org/10.1111/eth.13118).

15. Ahmed I, Faruque IA. 2022 High speed visual insect swarm tracker (hi-vista) used to identify the effects of confinement on individual insect flight. bioRxiv (doi:10.1101/2021.12.31.474665).

16. Faruque IA, Muijres FT, Macfarlane KM, Kehlenbeck A, Humbert JS. 2018 Identification of optimal feedback control rules from micro-quadrotor and insect flight trajectories. Biological cybernetics 112, 3, 165-179.

17. Faruque IA, Humbert JS. 2014 Wing motion transformation to evaluate aerodynamic coupling in flapping wing flight. Journal of theoretical biology $\mathbf{3 6 3}$, $198-204$.

18. Sun M. 2014 Insect flight dynamics: stability and control. Reviews of Modern Physics 86, 2, 615 .

19. Faruque I, Humbert JS. 2010 Dipteran insect flight dynamics. part 1 longitudinal motion about hover. Journal of theoretical biology 264, 2, 538-552.

20. Sherman A, Dickinson MH. 2004 Summation of visual and mechanosensory feedback in drosophila flight control. Journal of experimental biology 207, 1, $133-142$. 
21. Hedrick TL, Cheng B, Deng X. 2009 Wingbeat time and the scaling of passive rotational damping in flapping flight. Science 324, 5924, 252-255.

(doi:10.1126/science.1168431).

22. Faruque I, Humbert JS. 2010 Dipteran insect flight dynamics. part 2: lateral-directional motion about hover. Journal of theoretical biology 265, 3, 306-313.

23. Josephson RK, Malamud JG, Stokes DR. 2000 Asynchronous muscle: a primer. Journal of Experimental Biology 203, 18, 2713-2722.

24. Altshuler DL, Dickson WB, Vance JT, Roberts SP, Dickinson MH. 2005 Short-amplitude high-frequency wing strokes determine the aerodynamics of honeybee flight. Proceedings of the National Academy of Sciences 102, 50, $18213-18218$.

25. Vance J, Altshuler D, Dickson W, Dickinson M, Roberts S. 2014 Hovering flight in the honeybee apis mellifera : Kinematic mechanisms for varying aerodynamic forces. Physiological and biochemical zoology : PBZ 87, 870-81. (doi:10.1086/678955).

26. Humbert JS, Faruque I. 2011 Analysis of insect-inspired wingstroke kinematic perturbations for longitudinal control. Journal of Guidance, Control, and Dynamics 34, 2, 618-623.

27. Zheng L, Mittal R, Hedrick T. 2010 A search for optimal wing strokes in flapping flight: Can engineers improve upon nature? In: 28th AIAA Applied Aerodynamics Conference. (doi:10.2514/6.2010-4944).

28. Read TJG, Segre PS, Middleton KM, Altshuler DL. 2016 Hummingbirds control turning velocity using body orientation and turning radius using asymmetrical wingbeat kinematics. Journal of the Royal Society, Interface 13, 116. (doi:10.1098/rsif.2016.0110). 